\title{
Do Fitting Emotions Tell Us Anything About Well-Being?
}

James Fanciullo

Forthcoming in Utilitas

\begin{abstract}
In a recent paper in this journal, Tobias Fuchs has offered a 'working test' for well-being. According to this test, if it is fitting to feel compassion for a subject because they have some property, then the subject is badly off because they have that property. Since subjects of deception seem a fitting target for compassion, this test is said to imply that a number of important views, including hedonism, are false. I argue that this line of reasoning is mistaken: seems fitting does not imply is badly off. I suggest that Fuchs's test can tell us little about well-being that we do not already know; and ultimately, tests of the sort he proposes can yield little insight into the nature of well-being.
\end{abstract}

Theories of well-being or welfare essentially aim to tell us which things are basically good and bad for us_-or which things are the basic constituents of our well-being and ill-being. According to some theories, all and only experiences of a certain kind can directly contribute to our well-being. No doubt the most popular such theory is hedonism, which claims that all and only enjoyable experiences directly contribute to a subject's well-being, and all and only unpleasant experiences directly contribute to a subject's ill-being. ${ }^{1}$

While experiential theories like hedonism have been under attack for some time, ${ }^{2}$ this attack has recently become more pronounced. The problem for these theories is that they seem to predict

\footnotetext{
${ }^{1}$ For contemporary defenses of hedonism, see R. Crisp, Reasons and the Good (Oxford, 2006), ch. 4; F. Feldman, Pleasure and the Good Life (Oxford, 2004), ch. 4. Here I follow Crisp in formulating hedonism in terms of enjoyment, rather than pleasure, for reasons he notes. I also use 'unpleasant' rather than 'painful', for parallel reasons. An alternative experiential theory, 'experientialism', has also recently been defended by Richard Kraut, The Quality of Life: Aristotle Revised (Oxford, 2018).

2 See e.g. Plato, Philebus (Indianapolis, 1993); G. E. Moore, Principia Ethica (Cambridge, 1903), ch. 3; W. D. Ross, The Right and the Good (Oxford, 1930), ch. 5.
} 
that nothing external to a subject's experiences should have any direct influence on the subject's well-being. After all, if well-being consists in (e.g.) enjoyable experience, then facts about the person's life that do not directly impact their enjoyable or unpleasant experiences should be irrelevant to their well-being. Recently, however, it has been argued that facts of this kind can indeed directly impact a subject's well-being. ${ }^{3}$ One such argument has come from Tobias Fuchs, who appeals to the following case:

Imagine two women, both under the impression of being in a loving marriage. Their lives are identical apart from the fact that one of them [call her Jess] has the right impression, while the other one [Ingrid] is mistaken — she is being lied to and cheated on by her husband. ${ }^{4}$

Theories like hedonism appear to imply that Jess and Ingrid should seem to us to be equally well off. That's because hedonism claims that well-being consists in a certain kind of experience, and Jess and Ingrid each have the same experiences. The theory therefore implies that the two have equal levels of well-being; and so, the hope for the hedonist will no doubt be that our intuitions accord with this implication.

The problem, of course, is that our intuitions about the case are not as hedonism seems to predict: Jess intuitively seems better off than Ingrid. ${ }^{5}$ Since Jess seems better off than Ingrid merely

\footnotetext{
${ }^{3}$ Most recently, see e.g. T. Fuchs, 'A Working Test for Well-Being', Utilitas 30 (2018), pp. 129-42; E. Lin, 'Pluralism about Well-Being', Philosophical Perspectives 28 (2014), pp. 127-54; E. Lin, 'How to Use the Experience Machine', Utilitas 28 (2016), pp. 314-32; C. Stevenson, 'Experience Machines, Conflicting Intuitions and the Bipartite Characterization of Well-being', Utilitas 30 (2018), pp. 383-98. These arguments are of course inspired by R. Nozick, Anarchy, State, and Utopia (New York, 1974), pp. 42-5. For 'just a sample' of people who endorse Nozick's discussion as a refutation of hedonism - a sample listing twenty-eight examples— see D. Weijers, 'Nozick's Experience Machine Is Dead, Long Live the Experience Machine!', Philosophical Psychology 27 (2014), pp. 513-35, at 530.

${ }^{4}$ Fuchs, 'Working Test', p. 139.

${ }^{5}$ Nonetheless, some have appealed to experimental data in attempt to show that our intuitions of this sort —and specifically those relating to Nozick's experience machine-tell us little about the truth or falsity of hedonism. See e.g. F. De Brigard, 'If You Like It, Does It Matter If It's Real?', Philosophical Psychology 23 (2010), pp. 43-57; Weijers, 'Nozick’s Experience Machine'.
} 
in virtue of some fact about her life that does not directly impact her enjoyable experiences, the veracity of our intuition here seems to conflict with the truth of hedonism. And so, either our intuition or hedonism must be given up. While this argument is simple enough, it is also quite powerful. It leaves the hedonist in the uncomfortable position of having to 'bite the bullet', either by suggesting that the intuition is for some reason misleading, or claiming that they themselves simply do not have the same intuition as many of us.

Regardless of how plausible we take these potential responses of the hedonist to be, Fuchs has offered what he takes to be a principled argument against them. Indeed, Fuchs offers a 'working test' for well-being, which aims to provide an independent standard for testing when exactly a subject's well-being has been impacted. The idea behind the test is that, when we feel compassion for someone in virtue of some fact about them, and our compassion also meets a certain standard of correctness — when it is also the 'right' sort of emotion to feel — the subject's well-being is negatively impacted in virtue of that fact about them. So since it seems right, for example, to feel compassion for Ingrid in virtue of her being deceived, the test apparently tells us that Ingrid is worse off in virtue of her being deceived. Given it reaches this conclusion by appealing only to facts about the correctness of compassion, and not to any claims or intuitions about well-being, Fuchs's test seems to provide an independent and conclusive way of both testing for changes in well-being, and refuting theories like hedonism. My aim in this paper is to show that, in fact, tests of the sort Fuchs proposes can tell us little more than what we already know about well-being, and certainly cannot teach us that experiential theories are false.

\section{Fuchs's test and its significance}

The general idea behind Fuchs's test, I have said, is that if compassion meets some standard of correctness - if it is the 'right' sort of emotion to feel—then we are free to conclude that the 
relevant subject is in some respect badly off. But what exactly is the relevant standard of correctness here that our compassion must meet?

The standard of correctness here is the emotion's fittingness. Emotions are typically taken to have not only affective and motivational components, but evaluative components as well. To see this, consider fear. Fear is certainly marked by a certain affect, and also disposes those who feel it to perform certain actions. Yet fear also has an evaluative component: it involves apprehending the target of that fear, or the thing at which the fear is directed, as instantiating a certain evaluative property — a property that is sometimes called the emotion's formal object. ${ }^{6}$ In the case of fear, the formal object is the property of being dangerous (or being a danger to the fearer). This is evidenced by the fact that it would not make sense for me to feel fear if I did not take the target of that fear to be dangerous: if I did not experience the target as dangerous, there would be nothing to make my experience of fear intelligible. We can then say that my emotion 'makes sense' just in case I see the target as possessing the emotion's formal object. Importantly, however, a subject's emotion can sometimes make sense, even though we do not think the emotion is correct or fitting. If, for example, I know that a nearby spider is harmless, yet I fear it just the same, we should say that although my emotion makes sense, because I do see the spider as being dangerous, it is not correct or fitting, because the spider is not in fact a danger to me. If, in contrast, I am fearful of a nearby wild tiger, which I know is likely to attack me, my fear not only makes sense, because I see the tiger as being dangerous, but is correct or fitting, because the tiger is in fact a danger to me. More generally: an emotion is fitting if and only if the target of the emotion in fact has the property that serves as the emotion's formal object. ${ }^{7}$

\footnotetext{
${ }^{6}$ See J. A. Deonna and F. Teroni, The Emotions: A Philosophical Introduction (New York, 2012), p. 41; Fuchs, 'Working 'Test', p. 135.

7 See J. D'Arms and D. Jacobson, 'The Moralistic Fallacy: On the "Appropriateness" of the Emotions', Philosophy and Phenomenological Research 61 (2000), pp. 65-90; Deonna and Teroni, Emotions.
} 
After outlining this notion of fittingness, Fuchs applies it to compassion. He notes that it does not make sense to feel compassion for someone whose life we take to be in all respects going well. If, for instance, your friend was by all accounts happy, healthy, wealthy, and wise, you may think it odd of me to report feeling compassion for her. Yet if I told you that she will soon be diagnosed with a serious illness, my compassion will seem perfectly in order. While this alone may not show that the property of being worse off is compassion's formal object, it at least suggests that a reduction in well-being is a necessary condition for compassion to be fitting. That is, it suggests that compassion's formal object, whatever it may be, must overlap with the property of being worse off at least to the extent that, whenever it is instantiated, the property of being worse off is instantiated as well. Together with the outlined notion of fittingness, this yields Fuchs's test for well-being:

If compassion for some person A is fitting in virtue of A's having (or lacking) some property P, then A's well-being is negatively affected by A's having (or lacking) that property $\mathrm{P} .{ }^{8}$

Since an emotion is fitting if and only if its target in fact has the property that serves as its formal object, it follows, so long as the fittingness of compassion requires that the target has the property of being worse off, that if compassion is fitting in virtue of the target's having or lacking some property, then the target of the compassion is worse off in virtue of their having or lacking that property. The fittingness of compassion is then a sufficient condition for welfare having been diminished.

Having established his test, Fuchs suggests a way of utilizing it. He writes:

We compare two lives, similar in all respects but that one has a property the other one lacks. Now we see whether compassion is fitting towards one of them, in virtue of having (or lacking) that property.

${ }^{8}$ Fuchs, 'Working Test', p. 137. 
If it is, we know that having (or lacking) that property is negatively affecting that person's wellbeing. ${ }^{9}$

The test can then be used to support our intuition that Ingrid is worse off than Jess. Since Ingrid is being cheated on by her husband and Jess is not, it follows, so long as Ingrid is the fitting target of compassion in virtue of being cheated on by her husband, that Ingrid is worse off than Jess in virtue of being cheated on by her husband. Consequently, it seems, the fact that a subject is deceived can negatively impact the subject's well-being.

As I've mentioned, Fuchs takes his test to provide us with an independent standard for changes in well-being whose authority cannot simply be dismissed by those whose theoretical commitments clash with its implications. That's because fittingness tracks the truth: an emotion's formal object must in fact be instantiated in order for the emotion to be fitting. Accordingly, so long as we establish that compassion would be fitting in some case, and that compassion's fittingness requires that its target is in some regard badly off, the claim that the target of the compassion is nevertheless no worse off is not a response that is available to the would-be objector. After all, by accepting that the compassion is fitting, we accept that the target is worse off. The verdicts of the test then cannot plausibly be refuted on the grounds of conflicting theoretical commitments.

Now, that may be right, but notice how Fuchs actually applies his test:

It seems that compassion is fitting towards [Ingrid], in virtue of her spouse's cheating on her and deceiving her. If we overheard a conversation in a diner of someone telling his friend about his sister being in Ingrid's situation, and he expressed compassion for his sister, surely we would not find this unintelligible — the stranger's compassion is perfectly fitting here. According to our compassion test,

\footnotetext{
${ }^{9}$ Fuchs, 'Working Test', p. 137.
} 
it follows that the woman is badly off in virtue of her being deceived and cheated on by her spouse. ${ }^{10}$

Here, Fuchs appeals to the fact that compassion for Ingrid seems fitting, and concludes on that basis that Ingrid is worse off. Notice, however, that the test says nothing about whether compassion seems fitting; only something about whether compassion is fitting. To be sure, many of us will share Fuchs's intuition that compassion seems fitting, but this intuition does not tell us that compassion is in fact fitting. It tells us only that compassion seems fitting. And, of course, it does not follow from the fact that compassion seems fitting that compassion is fitting-given it does not follow from the fact that a subject seems to have some property that the subject has that property-and so it does not follow from Fuchs's arguments that Ingrid's well-being is diminished.

The task for Fuchs, then, is to show that compassion for Ingrid is fitting. The problem here, however, is that we simply cannot know whether compassion for a subject is fitting without knowing whether the subject's well-being is diminished. To see why, return to the example of fear. In the case of the nearby tiger, we can conclude that my fear is fitting, because we know that the tiger is a threat to me. And, in the case of the nearby spider, we can conclude that my fear is not fitting, because we know that the spider is not a threat to me. But now consider a case where we're not sure whether the animal is a threat to me. Suppose there's a nearby dog, which gives me some reason to perceive it as a threat—say, that it appears to be of an 'aggressive' breed—and which I therefore fear, despite being unsure of how likely it is to attack. Since I see the dog as a threat, my emotion here surely makes sense. But is my emotion here also fitting? Well, of course, it depends. In particular, it depends on whether the dog is in fact a threat to me-on whether it is sufficiently likely to attack and harm me. If we do not know that it is in fact a threat to me, then we cannot conclude that my fear is fitting. The most we can conclude is that, given the dog appears to me to be of an

${ }^{10}$ Fuchs, 'Working Test', p. 139. 
aggressive breed, there is at least one reason for thinking that my fear may be fitting.

In the same way, I think, the most we can conclude about Fuchs's case is that, given Ingrid appears to us to be worse off, there is at least one reason for thinking that compassion for her may be fitting. After all, whether compassion is fitting depends on whether the target's welfare is diminished; and so, in order to know whether compassion for someone is fitting, we must know whether their welfare is diminished. Yet how could we know whether someone's welfare is diminished, without the true theory of welfare? The answer, outside of any case where it is already indisputable that welfare is or isn't diminished, is that we cannot. If we do not already know whether a subject's well-being is diminished in some case-if it is not a case, such as one involving sustained and brutal torture, where it could not plausibly be denied that the subject's welfare is diminishedthen we cannot presently know whether compassion for the subject is fitting, because we do not know whether the subject's well-being is diminished. This means that, so long as we do not know what well-being consists in, any case where we do not already know whether well-being is diminished will also be a case where we do not know whether compassion is fitting. And so, since we do not know what well-being consists in, and since we do not already know, in Fuchs's case, whether Ingrid's well-being is diminished, it follows that we do not know whether compassion for her is fitting. We then cannot conclude that compassion for her is fitting, and so cannot employ Fuchs's test here. The most we can conclude is that, given Ingrid seems to us to be worse off, there is at least one reason for thinking that compassion for her may be fitting. Just as in the case of my fear of the dog, we cannot know whether our emotion is fitting without knowing whether the target has the relevant property.

(It is worth briefly mentioning that this epistemological limitation will apply, for analogous reasons, to any emotion whose fittingness requires some change in its target's well-being. So long as we do not know either what well-being consists in, or whether the target's well-being has been 
changed in the relevant way, we cannot know whether the emotion is fitting. I will return to this point below.)

Importantly, in making this argument, I do not mean to appeal to any kind of extreme skepticism about what we can or do know about well-being. As I've suggested, I think there are at least some cases, such as those involving brutal torture, where we do know that a subject's wellbeing is diminished, despite our not knowing what well-being consists in. As it happens, I think these are also just the cases where we can surely utilize Fuchs's test: since we know that welfare is diminished, we can conclude that compassion is fitting, and so can conclude via Fuchs's test that welfare is diminished. As this exercise shows, however, the cases where we can surely utilize his test are just those where the test is needed least. Since we cannot know whether compassion is fitting without knowing whether welfare is diminished, we cannot confidently apply Fuchs's test without already knowing the very conclusion that the test is meant to help us reach. Seen in this way, Fuchs's test and its implications for the debate over the nature of well-being are likely to seem far less significant than Fuchs sometimes suggests them to be. At the very least, it seems clear that the test does not prove to us that experiential theories like hedonism are false, since the cases that would allow the test to prove this are just those where we do not know whether compassion is fitting. But, even if that is right, it may be objected that the test still provides some independent reason for rejecting these views. After all, in cases like Ingrid's, many of us will agree that compassion for Ingrid seems fitting. And, it may be argued, while this may not prove the claim that compassion for Ingrid is fitting, it at least justifies that claim-it allows us to justifiably believe it, given our emotion does not seem subject to any defeaters, such as hallucination or drunkenness. In that case, it seems we are also justified in claiming that Ingrid's welfare is diminished, since compassion is fitting only if a subject's welfare is diminished. The objection, then, is that even if Fuchs's test faces the epistemological limitation I discussed, the fact that compassion for Ingrid 
seems fitting still provides at least some reason for thinking that she is worse off, and so at least some reason for thinking that theories like hedonism are false.

I'm willing to concede that compassion for Ingrid seems fitting, and compassion for Jess does not. I'm also willing to concede that this provides some reason for thinking that Ingrid is (or, at least, will be) worse off than Jess. What I am not willing to concede, though, is that any of this provides a reason for thinking that hedonism is false. In fact, I think it is more likely to provide a reason for thinking that we have misidentified the property in virtue of which compassion for Ingrid seems fitting. Since Ingrid and Jess are in loving and happy marriages, being cheated on would almost certainly lead either of them to many, very unpleasant experiences. But because only Ingrid is being cheated on, only Ingrid is overwhelmingly likely to experience this great deal of unpleasantness in the future. As I see it, it seems far more plausible that it is in virtue of this fact that compassion for Ingrid seems fitting, and compassion for Jess does not, than it seems plausible that being deceived or cheated on would explain this difference.

Perhaps you disagree. Then consider a slightly modified version of the case. Suppose Ingrid and Jess's marriages are instead not happy, and that if either of them were to learn of their partner's infidelity, it would not lead to any unpleasantness at all. In fact, it would make them happy: they would see it as a relief, and would eventually look back on it positively. Would compassion for Ingrid, whose partner is in fact cheating on her, still seem fitting in this case? As I see it, while compassion might seem fitting in virtue of her being in an unhappy marriage (given the likely attendant unpleasantness), it would not seem fitting in virtue of her being deceived or cheated on. Since she would see it as a relief upon finding out about the deception, it would not cause her to experience any immediate unpleasantness; and since she would look back on it positively, it would not cause her to experience any unpleasantness in the long term. Even if you disagree that compassion does not seem fitting in virtue of Ingrid's being deceived or cheated on here, though, 
I'm confident you'll agree that it seems far less fitting in this case than it does when she is in a happy marriage. Yet, in each case, she is equally cheated on and deceived; and so, if these are indeed ultimately the properties in virtue of which compassion for her is fitting in one case, compassion for her should seem equally fitting in the other case. That is, if the fact that ultimately explains why compassion for Ingrid is fitting — and thus why she is worse off-when her marriage is happy is that she has the property of being deceived, then compassion for Ingrid should seem equally fitting when her marriage is not happy, because that fact applies to her just the same. Since compassion for Ingrid does not seem equally fitting in the cases, it seems considerably less plausible that this is ultimately the property in virtue of which compassion for her is fitting in either case. And if this is not the property in virtue of which compassion for Ingrid is fitting, then, while the fact that compassion for Ingrid seems fitting and compassion for Jess does not may provide some reason for thinking that Ingrid is (or, at least, will be) worse off than Jess, that fact does not also provide a reason for thinking that deception, or something outside of Ingrid and Jess's experiences, is what ultimately explains this difference in well-being. Contrary to the objector's suggestion, then, our intuitions about compassion's fittingness do not provide a reason for thinking experiential theories like hedonism are false. In fact, given that the case where compassion for Ingrid seems more fitting also happens to be the one where she seems more likely to experience more unpleasantness, and the case where compassion for her seems less fitting also happens to be the one where she seems less likely to experience any unpleasantness, the hedonist (and experientialist more generally) seems particularly well-positioned to plausibly explain why compassion seems more or less fitting in the cases. ${ }^{11}$

As my arguments here illustrate, our intuitions about compassion's fittingness are in no

\footnotetext{
${ }^{11}$ It's worth mentioning, however briefly, that I think the most plausible version of this explanation will appeal to a proposed gap between the basic constituents of our well-being, on the one hand, and our intuitions about changes in well-being, on the other. Such a gap would make room for claiming that while well-being consists in (e.g.) enjoyable experience, our intuitions about changes in well-being track present and future such experience. In that case, the fact that a subject like Ingrid seems worse off may not entail that their well-being has diminished—at least, not yet.
} 
better position to tell us either when changes in well-being have occurred, or which properties ultimately explain these changes, than our everyday intuitions about changes in well-being. While these intuitions about compassion's fittingness may reinforce what our intuitions about changes in well-being can already tell us, we should be careful not to overstate their significance. They surely do not provide conclusive evidence of anything that would significantly advance the debate over the nature of well-being (such as the falsity of experiential theories), and indeed are just as likely to mislead us with respect to determining which things are basically good and bad for us as our everyday intuitions about changes in well-being. Accordingly, if our aim in this debate is to determine which things are basically good and bad for us, appealing to the fittingness of compassion does not seem an especially promising way forward.

Really, these points apply to any putative 'test' for well-being that appeals to the fittingness of an emotion whose fittingness requires some change in its target's welfare. That's because, since fittingness tracks the truth, we will be unable to conclude that any such emotion is fitting without knowing whether the target's well-being has been impacted in the relevant way, leaving us to retreat to our intuitions about whether the target's well-being has been so impacted, and thus about whether the emotion is fitting, in any case where these things are not already known. While our intuitions here may provide some evidence for claiming that the target's well-being has been impacted, they may also provide misleading evidence about the ultimate explanation for that impact. Since the debate over the nature of well-being is essentially concerned with determining which things are basically good and bad for us_-or determining which things ultimately explain changes in well-being - these 'tests' therefore seem no better positioned to significantly advance the debate than appeals to our everyday intuitions about changes in well-being. I hope to have shown here that, while it may at times be tempting to think otherwise, significant progress in this debate still requires the difficult work of reasoning our way, via our intuitions, to the true theory of well-being. Fitting 
emotions can provide no shortcut. ${ }^{12}$

12 Many thanks to Richard Kraut, Doug Portmore, and two anonymous reviewers for extremely helpful comments and discussions. 